\title{
El Paradigma de las Neurociencias de la Educación y el Aprendizaje del Lenguaje Escrito: Una Experiencia de 60 Años
}

\section{The Paradigm of Educational Neuroscience and the Learning of the Written Language: Sixty Years After}

\author{
Luis Bravo \\ Pontificia Universidad Católica de Chile
}

\begin{abstract}
El objetivo de este artículo es presentar la experiencia del autor sobre los aportes de las neurociencias y de la psicología cognitiva a la investigación del aprendizaje del lenguaje escrito desde la perspectiva del modelo de paradigmas científicos propuesto por el filósofo Thomas Kuhn para la historia de las ciencias. Se describen las investigaciones y el avance de la psicología cognitiva y de las neurociencias de la educación en el aprendizaje del lenguaje escrito durante 60 años. Los conocimientos derivados de las neurociencias de la educación y fundamentadas en neuroimágenes muestran una estrecha relación entre los procesos cognitivos cerebrales y la psicopedagogía del aprendizaje escolar.
\end{abstract}

Palabras clave: paradigma, lenguaje escrito, neurociencias, psicología cognitiva, dislexia

\begin{abstract}
The aim of this article is to present the author's experience regarding the contributions of neurosciences and cognitive psychology to research on the learning of written language from the perspective of the model of scientific paradigms, advanced by the philosopher Thomas Kuhn for the study of the history of science. The article describes the research and the progress made over the last 60 years in the field of cognitive psychology and educational neuroscience with respect to the learning of written language. The knowledge derived from educational neuroscience, based on neuroimaging, reveals a close connection between cognitive processes in the brain and the educational psychology of school learning.
\end{abstract}

Keywords: paradigm, written language, neurosciences, cognitive psychology, dyslexia

La historia de las ciencias psicológicas se remonta a la filosofía de las ciencias (Geymonat, 1970/1984; Woodward \& Ash, 1982). Esta dependencia original de la filosofía motiva con frecuencia a los psicólogos a asumir una postura epistemológica sobre la mente, la conciencia y el cerebro. Hernán Larraín me expresó en una carta personal de enero de 1956 que "El método del psicólogo ha de ser observación. (...) Observar es intuir, es penetrar el sentido de las cosas, llegar a su inteligibilidad descubriendo la huella del espíritu en la materia y en los hechos." (carta citada en Bravo, 2013, p. 128). Este método fenomenológico permite integrar la experiencia psicológica clínica con la experimental de la neurología. Larraín coincide con el psicólogo psicoanalista belga Vergote (1992), quien escribió que el crecimiento corporal y mental producen "una autoconstitución de la persona" (p. 174). "El sujeto se auto construye activamente, interiorizando lo que recibe del mundo y de la cultura" (p. 175) y el desarrollo de las personas es resultante de "una auto-transformación" que se inicia en el cerebro durante la infancia. Agrega que el origen que tienen en el desarrollo personal los "procesos psicológicos impide separar la naturaleza biológica y la cultural" (p. 177). Para Vigotski, el proceso mediador entre ambos se produce en el lenguaje y en el significado de las palabras y propuso el término neuro génesis para las modificaciones que recibe el cerebro por efecto de sus conexiones con el medio ambiente (del Río \& Álvarez, 2017).

\section{El Paradigma de las Neurociencias de la Educación}

Reid Lyon (1996) describió la neuropsicología del desarrollo como una subespecialidad científica y clínica que "combina el estudio del desarrollo cerebral con el estudio del desarrollo de la conducta" (p. 15). Mediante investigaciones y metodologías clínicas, es posible identificar los cambios que se producen entre la actividad del cerebro, el lenguaje y la conducta durante la infancia y la adolescencia. Es una relación que aparece en

Luis Bravo Valdivieso, Escuela de Psicología, Pontificia Universidad Católica de Chile, Santiago, ChileLa correspondencia relativa a este artículo debe ser dirigida a Luis Bravo Valdivieso, Escuela de Psicología, Pontificia Universidad Católica de Chile, Avda. Vicuña Mackenna 4860, Macul, Santiago, Chile. E-mail: abravov@uc.cl 
continuo cambio y muestran la incidencia que tienen los estímulos y ambientes educativos en el desarrollo cerebral.

Sin embargo, la experiencia psicopedagógica muestra que la trascripción de los conocimientos neuropsicológicos cuantitativos a las estrategias psicopedagógicas cognitivas y a los procesos cualitativos del lenguaje y del pensamiento es un proceso complejo, por la dificultad de encontrar las relaciones concretas entre el funcionamiento sináptico del cerebro y los proceso cognitivos (Bruer, 1997).

Damasio (2010), en su libro sobre el cerebro, considera que "aprender y generar memoria es simplemente el proceso de tallar, modelar, dar forma, hacer y rehacer los diagramas de conexión de nuestro cerebro" (p. 446). Una vía para entender cómo interviene el cerebro es investigar las conexiones entre sus diferentes regiones, lo que "no es tarea sencilla, ya que los diagramas de conexión experimentan considerables cambios a lo largo del proceso de desarrollo" (Damasio, 2010, p. 446). Estas modificaciones constituyen un objetivo del proceso educacional y ellas han sido confirmadas en las investigaciones mediante neuroimágenes durante el aprendizaje del lenguaje escrito y de las matemáticas, lo cual ha acercado a las ciencias neurológicas y las psicológicas con la pedagogía y permite establecer un nuevo paradigma de investigación denominado neurociencias de la educación, aplicable a la psicopedagogía. La originalidad de este aporte de las neurociencias a la psicopedagogía ha sido investigar los puntos de contactos y de interacción entre la biología del sistema nervioso central y los estímulos culturales, verbales y emocionales con las estrategias pedagógicas (Szűcs \& Goswami, 2007).

Mi objetivo como psicólogo clínico y educacional es describir mi percepción sobre la psicopedagogía del aprendizaje del lenguaje escrito, como un paradigma de investigación que integra a la neuropsicología, la psicología cognitiva y la lingüística con la educación.

He aplicado en él el término aprendizaje del lenguaje escrito, en vez de enseñar o aprender a leer y escribir. Esta modificación no es solamente semántica, sino epistemológica. Los niños que aprenden un lenguaje escrito no solamente deben reconocer su alfabeto o la información contenida en las palabras, sino aprender a reconocer la estructura de la lengua y a comprender cualquiera expresión de otro idioma. El concepto de lenguaje escrito implica ampliar la capacidad de pensar y de aplicar a la escritura ortográfica, con lo cual se supera la acción pedagógica de enseñar a leer hacia una metacognición (Bravo Valdivieso, 2016).

\section{La Neuropsicología y las Dislexias}

En 1960 comencé a trabajar en clínica y estudiar los problemas psicológicos de los niños que no aprendían el lenguaje escrito, para lo cual tuve que iniciarme en la neuropsicología e integrarla con la psicopedagogía. Recién se conocían en Chile las dislexias y su relación con el concepto de learning disabilities, propuesto por Kirk (1962), que entonces traduje como trastornos del aprendizaje (Bravo, 1990). El término dislexias era antiguo en Europa. Había sido utilizado por primera vez en 1887 en Alemania para describir un trastorno severo para leer por posibles causas neurológicas o visuales y, en 1896, Pringle Morgan le dio en Inglaterra el nombre de ceguera verbal congénita. En Estados Unidos, Orton en 1925 explicó esta dificultad como efecto de una deficiencia entre la corteza visual y el área de asociación cerebral, proponiendo una educación especial (investigaciones descritas en Bravo Valdivieso, 2011). De Latinoamérica conocí sobre las dislexias las publicaciones argentinas de Quirós y Della Cella (1965) y de Azcoaga (1979) y en Chile, las publicaciones de Olea (1966) y de Condemarín y Blomquist (1970).

La interrogante sobre las dificultades del aprendizaje de la lectura en muchos niños originaron mi primera investigación, que fue publicada en un pequeño libro sobre la disfunción cerebral mínima, mínima, donde describo mi experiencia clínica en 50 niños del Hospital Calvo Mackenna de Santiago, Chile, que no aprendían a leer (Bravo Valdivieso, 1973). Mi metodología fue psicométrica (WISC, Test de Bender, Rorschach), además de entrevistas clínicas, y los contrasté con un grupo control. No teníamos otros instrumentos de evaluación y diagnóstico. Ese libro tuvo tres ediciones, una de ellas prologada por el Premio Nacional de Ciencias, profesor Héctor Croxatto.

La gran cantidad de consultas por trastornos del aprendizaje de la lectura me motivó a seguir investigando y a publicar en diversas revistas las características psicológicas de los niños repitentes de cursos o desertores del sistema escolar por sus dificultades para leer. Lo hice en colaboración con otros psicólogos. Encontramos un alto porcentaje de escolares intelectualmente "normales" que presentaban trastornos en el rendimiento y alta incidencia de problemas de aprendizaje y trastornos psiquiátrico infantiles (Bravo \& Salas, 1972). Como consecuencia, quise crear conciencia pública de este desconocido problema educacional y clínico, 
calculando el costo que tenían las repitencias de los primeros años para la educación pública en Chile. Llegué a la pavorosa cifra que era equivalente al $10 \%$ del presupuesto nacional en educación primaria. Con esas cifras, conseguí que se publicara este estudio en la página editorial del periódico El Mercurio el 8 de mayo de 1971 y el Gobierno conociera este problema. Luego, don Juan Gómez Millas se interesó y me pidió que publicara un libro sobre la dislexia para la colección de libros del Consejo de Rectores (Bravo, 1980).

En 1979 la Revista Latinoamericana de Psicología me pidió editar un número especial sobre Psicología Educacional (Vol. $11 \mathrm{~N}^{\circ}$ 2) que reuniera investigaciones latinoamericanas. Concentré este número en las dificultades del aprendizaje escolar. Colaboraron en él Condemarín, Scagliotti, Álvarez, Heussler, Orellana, Chadwick, Tarky y Milicic de Chile y Sabogal, Molina y MacLean de Colombia y Guatemala.

Investigamos en las escuelas públicas la influencia de la maduración e integración de las funciones cognitivas cerebrales sobre el aprendizaje lector (Bravo, 1982), el pronóstico lector de los disléxicos (Bravo Valdivieso, 1978), los errores específicos en la lectura (Bravo, 1981a; Bravo Valdivieso \& Pinto, 1980; BravoValdivieso \& Pinto Guevara, 1984) y la disortografía disléxica (Bravo, Bermeosolo \& Pinto, 1987). Mediante el WISC-R, estudiamos las diferencias neuropsicológicas entre los niños con retardo lector (RL) severo y los lectores normales, pertenecientes al mismo y a diferentes niveles socioeconómicos (NSE; Bravo, Bermeosolo, Pinto, Oyarzo \& Morales, 1990; Bravo-Valdivieso, 1995). Estas investigaciones mostraron que las pruebas que discriminaban entre los niños con y sin RL de distintos NSE eran diferentes. Comparativamente, los niños con RL de NSE bajo presentaban mayor déficit en los procesos verbales. Preferían utilizar la vía visualverbal o logográfica para leer. Esto significa que tenían "menor techo" para la comprensión lectora. Ambos grupos socioeconómicos con RL mostraron deficiencias en los procesos de conciencia fonológica al ser comparados con lectores normales de un mismo nivel. Este resultado nos confirmó que los déficit en conciencia fonológica eran comunes a las dislexias, con independencia del NSE.

Posteriormente, investigamos la evolución cognitiva de estos niños y la posibilidad de predecir su futuro aprendizaje desde cuando iniciaban su escolaridad (Bravo, 1999; Bravo-Valdivieso, 1995; Bravo, Villalón \& Orellana, 2001, 2003; Bravo Valdivieso, Villalón \& Orellana, 2004, 2006a, 2006b). Para este trabajo elaboramos pruebas experimentales que aplicábamos luego de estudiar su confiabilidad y validez (Bravo, 2013; Bravo Valdivieso \& Pinto Guevara, 1987; Orellana, 1995).

Una investigación en 400 niños de seis años de primer año básico nos mostró que los niños pre escolares tenían un umbral lector y que el bajo rendimiento inicial se produce cuando no han desarrollado algunos procesos cognitivos y psicolingüísticos. Además, el déficit inicial tenía un efecto lector posterior que perturbaba su aprendizaje en los años siguientes (Bravo et al., 2001). El umbral lector estaba dado por el conocimiento de letras y de nombres escritos, mayor desarrollo de la conciencia fonológica y habilidad para determinar analogías verbales. Los niños lectores deficientes tenían un rendimiento inferior para identificar los fonemas de las palabras, segmentar pseudo palabras, encontrar analogías verbales, reconocer nombres escritos o el nombre de las letras. El retraso inicial se mantuvo en los cursos superiores (Bravo Valdivieso, 2011).

La importancia predictiva que tuvieron las pruebas de conciencia fonológica sobre la lectura nos confirmó que es el fundamento para su aprendizaje (Alegría, 1991, 2006; Vellutino, 1979). Culminamos las investigaciones con un seguimiento de 227 niños desde primer año básico, durante cuatro años. Fueron evaluados al iniciar su escolaridad con diversas pruebas psicolingüísticas y de lectura. Calculamos la variación de la lectura entre los diferentes cursos, mediante pre-test y post-test, que nos permitieron efectuar una matriz de correlaciones y de regresión stepwise. Los resultados confirmaron nuevamente que el conocimiento previo de letras y sílabas y la conciencia fonológica eran los mejores predictores de la lectura (Bravo Valdivieso et al., 2006a, 2006b).

Estas investigaciones las efectuamos con una metodología psicométrica, con análisis factorial, correlaciones, análisis de varianzas o análisis discriminante. Los resultados de la mayor parte de ellas aparecen en el libro Lectura Inicial y Psicología Cognitiva (Bravo Valdivieso, 2013).

También empecé a estudiar las investigaciones internacionales, que me condujeron a las neurociencias. Comencé por leer a Luria $(1966,1973)$ y, más tarde, conocí las investigaciones de Benton (1971) y Galaburda (1989) sobre el cerebro de los disléxicos.

Los temas del lenguaje y del pensamiento para el aprendizaje de la lectura los estudié en Vygotski (1934/1995) y en Piaget (1968) y, en relación con las dificultades para el lenguaje escrito, encontré como modelo las investigaciones de Vellutino (1979), Alegría (1991) y Azcoaga (1979). Me abrieron nuevos 
horizontes, modificando las hipótesis que las atribuían a déficit intelectuales, socioculturales o deficiencias perceptivo-visuales.

Posteriormente, tuve un intercambio personal con investigadores norteamericanos y europeos en la Academia Internacional de Investigación de las Dificultades del Aprendizaje, creada en Estados Unidos por el profesor Cruickshank. Él me invitó a participar en ella, lo que me permitió conocer personalmente a los investigadores en neurociencias.

En 1981 recibí una invitación del profesor Tarnopol, especialista en dificultades del aprendizaje en California (California Association for Neurologically Handicapped Children), para colaborar en un libro internacional comparativo sobre la educación especial de las dificultades del aprendizaje, describiendo la realidad de 23 países, hecha por especialistas de cada uno de ellos (Tarnopol \& Tarnopol, 1981). En lo referente a Chile, describí la relación entre las dificultades del aprendizaje escolar y el bajo NSE de la mayoría de los escolares de establecimientos públicos, que en Estados Unidos eran denominados subcultural minorities, y a los que atribuían el retardo mental y el fracaso escolar. Expresé que en Latinoamérica y en Chile los niños de pobreza eran una mayoría y que no podía atribuirse a la pobreza todas las dificultades del aprendizaje, sino también a insuficiencias pedagógicas (Bravo, 1981b).

En esos años empezó a investigarse con profundidad la interacción social y emocional en el desarrollo cerebral y en una Editorial del Journal of Child Psychology and Psychiatry Leckman y March (2011) expresaron que durante la formación de la neocorteza humana en el útero, además de los procesos genéticos que determinan el desarrollo de la neo corteza, la influencia del ambiente y de las relaciones del niño "pueden tener efectos directos y permanentes en el desarrollo del cerebro del niño y en su conducta" (p. 333). De esta manera, señalaron que hay un puente entre el desarrollo biológico genético y la influencia verbal emocional en el desarrollo cerebral.

Las investigaciones que conocí sobre el aprendizaje del lenguaje escrito y de las dislexias desde las perspectivas psicológicas cognitivas, psicolingüísticas y neurológicas me mostraron que ellas en conjunto podían constituir un paradigma científico.

El concepto de paradigma en filosofía de las ciencias, propuesto por Kuhn (1996), me facilitó integrar los aportes de las diversas investigaciones sobre las neurociencias y psicología de la educación. Kuhn propuso el concepto de paradigma para representar una manera de pensar y de actuar común a una comunidad científica. Expresó que "una comunidad científica está compuesta por hombres que comparten un paradigma" y tienen objetivos comunes (Kuhn, 1996, p. 176). En ciencias los nuevos paradigmas, con sus fundamentos teóricos y aplicaciones empíricas, integran los conocimientos existentes, solucionando crisis producidas por interrogantes que no han sido todavía respondidas por los investigadores (Kuhn, 1996). De esta manera, la emergencia de un paradigma afecta la estructura del conocimiento y puede ser un nuevo punto de partida para investigar problemas no resueltos.

Las investigaciones sobre el aprendizaje de la lengua escrita y las dislexias nos muestran una historia de pensamiento científico, que culminó en las neurociencias de la educación. La integración interdisciplinaria de los conocimientos de la psicología cognitiva, la neurología y la psicopedagogía fue un proceso lento, perfeccionado con la aparición de las neuroimágenes cerebrales. Ellas produjeron una eclosión de conocimientos que modificaron radicalmente el enfoque del aprendizaje escolar y de la convergencia de la actividad cerebral con los procesos cognitivos del aprendizaje del lenguaje escrito (Bruer, 1997). Revolucionaron los conocimientos psicopedagógicos sobre los trastornos del aprendizaje de la lectura. La exploración cerebral visualizada en neuroimágenes permitió conocer mejor la relación entre la percepción visual ortográfica y la conciencia fonológica con la enseñanza de palabras escritas y explicar mejor el origen de las dislexias. Sin embargo, según Goswami (2004), si bien la contribución de las neurociencias a la educación es grande, todavía falta construir puentes entre ellas.

El paso de la etapa de pre-paradigmas educacionales sobre el aprendizaje del lenguaje escrito al paradigma de las neurociencias se dio con los descubrimientos de las nuevas metodologías de observación e investigación del cerebro in vivo, mediante las imágenes de resonancia magnética (IRM), los potenciales evocados y/o la tomografía de emisión de positrones (TEP). Su principal aporte a la educación fue descubrir los puntos de interacción entre la biología del sistema nervioso central y los estímulos pedagógicos culturales, especialmente del lenguaje. Las neuro imágenes son clave para el conocimiento psicológico cognitivo y verbal en la lectura y su relación con la actividad de las diferentes áreas cerebrales. Un punto clave en el desarrollo de este proceso fueron las investigaciones sobre las dislexias en diferentes idiomas (Bravo, 2014). 
Los principales aportes neuropsicológicos aparecieron en numerosas investigaciones: Bruer (1997), Dehaene y Cohen (2007), Fawcett y Nicolson (2007), Goswami (2014), Jiménez (2012), Shaywitz y Shaywitz (2008), Sigman, Peña, Goldin y Ribeiro (2014) y Simos et al. (2002, 2007), entre otros. Ellos confirman que el aprendizaje del lenguaje escrito depende de las conexiones entre distintas áreas cerebrales. Katzir y PareBlagoev (2006) confirmaron como modelo para integrar las investigaciones de las neurociencias cognitivas con la psicología educacional a Bruer (1997), quien consideró que la psicología cognitiva sería el lugar de encuentro (middle ground as a midpoint) entre las neurociencias y la educación. Bruer (1997) estima que "las neurociencias cognitivas aportan una ventana en tiempo real a las estructuras y funciones cerebrales" y "la comprensión de las relaciones entre las diferentes estructuras del cerebro y sus funciones pueden ayudar a los científicos a comprender cómo ellas se relacionan con el aprendizaje y el desarrollo" (pp. 53-54).

Un ejemplo de esta relación entre psicología cognitiva y neurología en el aprendizaje del lenguaje escrito son las investigaciones sobre la percepción visual de las letras y palabras, efectuadas por Dehaene, Cohen, Sigman y Vinckier (2005). Observaron que en la región occipito-temporal izquierda del cerebro se configura un área que denominaron "región de la forma visual de las palabras" que registra el lenguaje escrito (Dehaene, 2007, p. 96). Este hallazgo fue confirmado por Brem et al. (2010), quienes estiman que la adquisición de la lectura desarrolla a nivel neural una nueva red funcional, a medida que los niños aprenden a asociar los sonidos del lenguaje con las características ortográficas. Esta red neural está localizada en la corteza izquierda occipito-temporal y la denominaron visual word-form system, la que se activa en los lectores al percibir las palabras escritas (Brem et al., 2010).

El conocimiento de las neurociencias modificó mi perspectiva para el trabajo psicológico y clínico y en educación. Los nuevos conocimientos derivados de las IRM, que muestran que el aprendizaje del lenguaje escrito está asociados con el desarrollo cerebral y con las variaciones bioquímicas y bioeléctricas que se producen en el cerebro, confirman que el aprendizaje escolar depende de un proceso neuropsicológico y cognitivo. Las técnicas de TEP, que registran el paso de las sustancias radioactivas por la corriente sanguínea del cerebro, y la imaginería funcional reflejan las variaciones del flujo sanguíneo y la actividad cerebral (Dehaene, 2007; Rumsey, 1996; Shaywitz et al., 2004).

Las neurociencias abrieron una senda para las intervenciones psicopedagógicas en el contexto de un paradigma científico. Conviene recordar que Kuhn comenzó su obra resaltando la importancia que tiene la historia de las ciencias para la configuración de los paradigmas científicos (Kuhn, 1996) y el filósofo alemán de las ciencias Radnitzky (1985) escribió que toda investigación científica tiene "una dimensión histórica" y un progreso cognitivo que busca mejorar los conocimientos, expresando que "la ciencia tiene una historicidad específica" (p. 134).

\section{La Historicidad de las Neurociencias Educacionales}

Según el neuropsicólogo Azcoaga (1979), las neurociencias comenzaron en Rusia con una presentación de Vygotski en un Congreso de Psiconeurología en 1923, quien puso sus bases, al asociar el desarrollo cerebral con los procesos socioculturales y del lenguaje. Akhutina (2002) confirma que Vygotski relacionó las formas superiores del lenguaje y la cognición con los lóbulos frontales y con las zonas témporo-parieto-occipitales del cerebro.

La relación entre los trabajos de Vygotski y Luria sobre la neuropsicología fue estrecha y Akhutina (2002) considera que "en correspondencia con la idea de L. S. Vygotski, A. R. Luria construyó una nueva área de la psicología: la neuropsicología" (2002, p. 125) e introdujo un modelo de funcionamiento cerebral en bloques de localizaciones dinámicas, "que se relacionan con la organización cerebral de los procesos psicológicos y el papel del lenguaje en la formación de las acciones voluntarias" (Luria, 1962, citado en Akhutina, 2002, p. 125). Luria (1973) también definió la percepción como "un proceso activo de búsqueda de la información que distingue los rasgos esenciales de un objeto, comparando sus rasgos con todos los demás, que establece hipótesis apropiadas y luego las compara con los datos originados" (p. 21). Expresa que el proceso perceptivo "es activo" (p. 23) y se efectúa a partir de "códigos pre establecidos", en particular, el código del lenguaje. También analizó las funciones fonémicas diciendo que "la palabra es una matriz multidimensional compleja de diferentes claves y conexiones (auditiva, morfológica, léxica y semántica) y sabemos que en diferentes situaciones predomina una de esas conexiones" (Luria, 1973, p. 306). Estas expresiones de Luria confirman la interdependencia entre los procesos cerebrales y el aprendizaje del lenguaje escrito como una vía para conocer las dislexias (Bravo Valdivieso, 2017). 
En los aportes de Vygotski a la pedagogía está el concepto de zona de desarrollo próximo (ZDP) como un "andamio mental" de aproximación a un conocimiento nuevo. En el caso de la lectura, la conciencia fonológica sería una ZDP, pues, con la ayuda del profesor, los niños pueden reconocer claves para la pronunciación de los fonemas, y facilitar la integración de las claves ortográficas de la escritura con las claves fonémicas del lenguaje oral (Bravo Valdivieso, 2002). La correcta pronunciación de las palabras da una clave de su significado y "la aplicación de estrategias ortográficas que se apoyen en los procesos semánticos depende de la habilidad que hayan tenido los niños para manejar los fonemas" (Bravo Valdivieso, 2013, p. 69).

\section{Las Vías Neurológica y Psicológica en la Historia de las Neurociencias}

En un libro sobre la evolución del cerebro y la creación de la conciencia, Eccles, premio Nobel de medicina en 1963, dice que "la estructura profunda de la gramática se asimila a la micro-organización de las áreas lingüísticas del cerebro. Se puede pensar que el niño nace con un 'conocimiento' de la estructura profunda del lenguaje, la cual está codificada en la micro estructura de las áreas lingüísticas de la corteza cerebral, que se forman antes del nacimiento" (Eccles, 1992, p. 118). En consecuencia, se puede considerar que un objetivo de las investigaciones neuro educacionales es determinar las áreas cerebrales que procesan el lenguaje oral y escrito y encontrar respuesta al dilema ¿dónde están las principales diferencias cerebrales entre los niños que aprenden a leer y los que tienen dificultades? (Bravo, 2014).

En la historia de las investigaciones sobre la lectura y las dislexias, encontramos dos vías seminales: las investigaciones neurológicas y las psicológicas cognitivas. Ambas son convergentes entre el desarrollo del sistema nervioso central y el aprendizaje del lenguaje. Su objetivo puede centrarse en la interrogante ¿qué ocurre en el cerebro cuando el ser humano aprende a hablar y leer? y ¿cuáles son las áreas cerebrales que intervienen? Las respuestas a estas interrogantes se fueron configurando en el paradigma de las neurociencias de la educación en dos líneas de investigación: la neurológica y la psicológica.

Entre las investigaciones neurológicas, ha sido fundamental la de Geschwind y Galaburda (1985) y Galaburda (1989) sobre las diferencias anatómicas del cerebro disléxico. Sus investigaciones dieron origen a una serie de información sobre los procesos cerebrales subyacentes al lenguaje escrito y las diferencias entre los cerebros "normales" y el de los disléxicos. Geschwind y Galaburda encontraron anomalías anatómicas en la citoarquitectura de la corteza del hemisferio cerebral izquierdo de un joven con dislexia severa, especialmente en la región temporal: "esas anomalías eran resultado de alteraciones en la migración neuronal y/o en su configuración” (Geschwind \& Galaburda, 1985, p. 428). Al respecto, Geschwind (1979) había escrito previamente que al leer "el patrón visual (proveniente del córtex visual primario) es trasmitido al giro angular, el cual opera una transformación que activa en el área de Wernicke la forma auditiva de la palabra" (p. 132). Considera que "el área de Wernicke no sólo interviene en el hablar, sino que desempeña también un papel importante en la comprensión de la palabra hablada y en el leer y el escribir" (p. 132). Galaburda y Cestnick (2003) consideran que los disléxicos tienen trastornos en el procesamiento de los fonemas y encontraron anormalidades citoarquitectónicas en las redes neuronales temporales, originadas en el período fetal, además de mostrar una asimetría inter hemisférica.

Hynd, Marshall y Semrud-Clikeman (1991) revisaron estas investigaciones con IRM y de tomografía computarizada, confirmando a Galaburda y Cestnick (2003) en que en los lectores normales predomina una asimetría en el plano temporal (hemisferio izquierdo mayor que hemisferio derecho) y una asimetría frontal, en cambio, en los disléxicos, había simetría o una asimetría revertida entre ambos hemisferios. Encontraron una asociación entre esta simetría y una menor comprensión lectora.

Estas investigaciones cerebrales mostraron que los disléxicos tienen un sustrato anatómico menor que los lectores normales en las áreas de procesamiento del lenguaje, lo cual explicaría su menor habilidad para aprender a leer y es determinante para la velocidad de la lectura y para la memoria del texto. Además, encontraron en los sujetos disléxicos menor actividad de circulación sanguínea en la región temporal anterior izquierda, junto con mayor activación del área posterior derecha. Habib (1997) desarrolló este tema en un libro titulado Dislexia: Un Cerebro Singular, donde describe las principales diferencias de los cerebros de los disléxicos.

En las investigaciones psicológicas tienen relevancia las de Vellutino et al., efectuadas desde la perspectiva psicológica lingüística. Entre los años 1972 a 2004 desarrollaron una línea de investigación sobre las dislexias como déficit específico del lenguaje, caracterizado por la inhabilidad de los disléxicos para verbalizar la información ortográfica (Vellutino, 1979; Vellutino, Steger \& Kandel,1972). 
Pudimos profundizar las investigaciones de Vellutino con su venida a Chile en 1979, a quien invité con colaboración del Fondo de las Naciones Unidas para la Infancia. Su visita implicó un profundo cambio en nuestra percepción de las dificultades del aprendizaje lector. Nuestra perspectiva psicopedagógica varió desde las hipótesis viso-perceptiva y psicomotora a una psicolingüística-fonológica. Esta variación implicó un cambio en nuestras estrategias de investigación y tratamiento de los niños. El año 2004 Vellutino, Fletcher, Snowling y Scanlon publicaron un amplio artículo sobre las investigaciones en dislexias en los últimos 40 años. Ellos concluyen que son una alteración genérica causada por problemas en la codificación lingüística y originadas en un déficit en el procesamiento fonológico.

El conocimiento de esas investigaciones internacionales me confirmó la hipótesis de un nuevo paradigma que permite integrar mejor las investigaciones psicológicas, neurológicas y psicopedagógicas, confirmando que las dificultades del aprendizaje de la lectura no son producidas solamente por deficiencias educacionales o sociales, sino que tienen un origen cerebral. Este tema me motivó a escribir un libro, publicado en España, sobre el enfoque neuropsicológico de las dislexias (Bravo Valdivieso, 1985) y, luego, otro en Chile sobre el lenguaje y las dislexias (Bravo Valdivieso, 2011). En estos libros traté de integrar investigaciones internacionales con nuestras experiencias chilenas de investigaciones efectuadas en escuelas municipales durante varios años, siguiendo lo que llamamos la vía psicológica de la lectura. Un resultado que nos impresionaba fue que el $22 \%$ de los niños disléxicos disminuyó su CI verbal luego de tres años de escolaridad (Bravo, Bermeosolo \& Pinto, 1988). Igualmente, continuando con la metodología de seguimientos, seguimos cuatro años a un grupo de alumnos disléxicos desde segundo año básico (Bravo et al., 1987). Un resultado fue que "la predictividad del CI (WISC-R) es baja para la comprensión lectora" y que el grupo disléxico "mantuvo durante el período de seguimiento una estrategia lectora preferentemente perceptivo-visual-secuencial de comprensión del texto, debido probablemente a insuficiencias en los procesos verbales superiores" (p. 141).

Todas estas investigaciones me mostraron que la realidad psicológica de los escolares chilenos había que abordarla con una nueva perspectiva y crear un Departamento de Educación Especial en la Universidad Católica, para formar profesores de educación especial o psicopedagogos. En nuestra línea de trabajo, nos apoyamos en las investigaciones de Jesús Alegría en Bruselas (Alegría, 1991, 2006) y de Vellutino (1979), quienes relacionan el desarrollo del lenguaje oral con el aprendizaje del lenguaje escrito. En la parte pedagógica colaboraron M. Condemarín, M. Chadwick y E. Orellana, en la Neurológica, J. Cox., y en la psicológica, N. Milicic, J. Scagliotti y J. Bermeosolo. Produjimos un Simposio Internacional en 1979, con apoyo del Fondo de las Naciones Unidas para la Infancia, denominado "El Niño con Dificultades para Aprender".

\section{El Aporte de las Neuroimágenes}

Las neuroimágenes confirman los conocimientos adquiridos en neurociencias y psicología cognitiva. McCandliss (2010) mostró los cambios que se producen en las imágenes cerebrales cuando se enseña a los niños a diferenciar la pronunciación de diferentes letras y su relación ortográfica. Expresa que las experiencias educacionales se asocian con la actividad cerebral para aprender a asociar con fluidez las letras escritas con sus respectivos fonemas. La confluencia audio-visual aparece muy disminuida en los disléxicos, "indicando una dificultad potencial para integrar ambos sistemas neurales" (p. 8050). Este estudio confirmó el eventual déficit de conexión intra-cerebral propuesto por Paulesu et al. (1996), al observar en los disléxicos una carencia de sincronía en las imágenes temporo-parietales durante la lectura. Por su parte, Poldrak (2001) también afirmó que "en la dislexia el giro angular del cerebro está funcionalmente desconectado" (p. 217). Estas investigaciones mostraron que tanto el giro angular como el plano temporal izquierdo serían clave para la conversión de la percepción visual ortográfica de las letras en sus respectivos fonemas, lo que fue confirmado por Sandak, Mencl, Frost y Pugh (2004), luego de un período de tratamiento psicopedagógico.

Las neuroimágenes muestran la interacción, velocidad e intensidad de las trasmisiones sinápticas durante el proceso educacional y permiten comparar la actividad de la conciencia fonológica y de la conciencia visual-ortográfica, en su relación con el aprendizaje de la lectura y el pensamiento verbal, en diversas áreas cerebrales (Rumsey, 1996). Por su parte, Goswami (2014) confirma que las imágenes son importantes para diferenciar a los lectores normales de los disléxicos. Menciona diversos estudios que muestran que con el aprendizaje se configura una red lectora en el cerebro, que se lateraliza preferentemente al lado izquierdo occipito-temporal posterior. En este punto coincide con Dehaene (2007), quien afirmó que, como consecuencia de la lateralización izquierda, "aprender a leer cambia el cerebro" (p. 210). 
La conjunción de las investigaciones mencionadas me permitió conocer mejor la importancia de las vías psicolingüísticas para el aprendizaje de la lectura y las conexiones que fallan en el cerebro de los disléxicos. $\mathrm{Al}$ respecto, Willingham y Lloyd (2007) expresaron que el acercamiento entre el nivel psicológico y el nivel neurológico constituye "un constructo cognitivo" (p. 145) y que un proceso clave para la educación es el análisis de las imágenes de las interacciones entre las distintas áreas cerebrales. Consideran que los procesos verbales y cognitivos requieren modelos teóricos psicopedagógicos de enseñanza que no siempre es posible asociarlos con determinadas actividades cerebrales, pero que pueden configurarse en un paradigma.

Shaywitz y Shaywitz (2008) también mostraron que en los niños disléxicos habría una alteración específica en el sistema cerebral posterior que afecta el aprendizaje de la lectura. Sus neuroimágenes muestran alteraciones en las áreas izquierdas parieto-temporal y occipito-temporal. También mostraron que la intervención pedagógica intensiva produce una normalización en la organización cerebral de los disléxicos, de tal manera que los niños con dificultades para leer puedan llegar a ser lectores hábiles. Ozernov-Palchik y Gaab (2016) concuerdan en que la convergencia, durante décadas, de las investigaciones neuropsicológicas con el advenimiento de las tecnologías de neuroimágenes nos colocaron en el umbral de una mayor comprensión de los modelos cognitivos y conductuales de las dislexias y de su prevención.

Las investigaciones muestran que, aunque las dislexias dependan de anomalías genéticas y biológicas, el aprendizaje del lenguaje escrito también depende del medio socio familiar y escolar. Coinciden con Fletcher (2009), quien expresó que "aprender a leer, literalmente reescribe la organización del cerebro" (p. 504), pues aparece una secuencia de componentes neurales que se van integrando en complejidad creciente.

Para entender mejor esta perspectiva de evolución neurocognitiva, Willingham y Lloyd (2007) sugieren recurrir a una teoría de las representaciones mentales. Expresan que ellas habrían sido "la base de la revolución cognitiva en la psicología experimental durante los años 1950 y 1960" (p. 140) y sirven como bloques de la construcción cognitiva, pues derivan de la integración de representaciones y procesos neuropsicológicos. Los procesos educacionales obligan al cerebro a transformar en lenguaje los estímulos y experiencias para darles significado. En el caso del aprendizaje del lenguaje escrito, el cerebro asocia las imágenes visuales ortográficas con unidades fonémicas y léxicas del lenguaje oral, las que configuran una "gestalt fonográfica", que permite asociar la pronunciación de las palabras con su significado (Bravo Valdivieso, 2011, p. 148).

\section{Conclusiones}

Luego de 60 años de seguir y participar en las investigaciones sobre las dislexias, concuerdo con Katzir y Pare-Blagoev (2006) en que "la combinación de las neurociencias y de la educación representa una nueva frontera en la ciencia; la cual toma tiempo y trabajo para desarrollar nuestro conocimiento fundacional en este nuevo campo del conocimiento de la mente, el cerebro y la educación" (p. 54). Agregan que las neurociencias cognitivas proveen una ventana en tiempo real a las estructuras y funciones del cerebro, lo que favorece la comprensión del aprendizaje y del desarrollo. Esta integración de investigaciones con un objetivo determinado en el aprendizaje del lenguaje escrito constituye un nuevo paradigma en el sentido expresado por Kuhn (1996) en su filosofía de las ciencias. Willingham y Lloyd (2007) también expresan que, así como toda actividad mental induce una activación cerebral, en la "teoría de las dislexias se asocian los problemas lectores con problemas específicos del lenguaje" (p. 143) y el aprendizaje del lenguaje escrito es un resultado de la integración entre la actividad de áreas cerebrales con las condiciones pedagógicas socio culturales que obligan al niño a seguir un logaritmo de asimilación y acomodación al alfabeto escrito. Las investigaciones sobre las dislexias también permiten afirmar que el paradigma de las neurociencias cognitivas ha abierto una frontera en el conocimiento del lenguaje escrito y su asociación con los procesos cerebrales que pueden ser percibidos en las neuroimágenes. Ozernov-Palchik y Gaab (2016) consideran que esta convergencia durante décadas de las investigaciones de la conducta con las tecnologías de neuroimágenes nos han colocado en un umbral de mayor comprensión de los procesos cognitivos, conductuales y neurales y requiere una renovación permanente de las estrategias psicopedagógicas.

El estudio de la evolución histórica de la psicología cognitiva durante 60 años modificó profundamente mi percepción de psicólogo de algunos procesos educacionales y ha abierto horizontes para integrar el aprendizaje escolar con la actividad cerebral. Las investigaciones de imágenes in vivo confirman que el cerebro es la pieza maestra de la autoconstrucción cognitiva del aprendizaje, del lenguaje, de las percepciones y del pensamiento. La neuropsicología es una base para la psicopedagogía, pues muestra cuáles son los procesos neurocognitivos subyacentes al aprendizaje y da claves para el tratamiento de las dislexias, 
tendiendo puentes con nuevas metodologías psicopedagógicas adecuadas para los niños con dificultades para aprender (Bravo Valdivieso, 2017). Entre sus principales aportes a la educación, están las investigaciones del desarrollo de los procesos cognitivos y verbales infantiles, del lenguaje, la memoria, el pensamiento, la conciencia fonológica, la percepción visual, las inteligencias múltiples y los procesos ejecutivos cerebrales, lo que ha permitido descubrir mejor las bases biológicas del aprendizaje escolar y las causas de algunos trastornos para aprender. Ellos, luego de 60 años, confirman la configuración de un paradigma científico, que históricamente partió de la neurología y de la psicología clínica.

Finalmente, coincido con Maurer y McBride (2017), quienes expresan que aprender a leer y escribir requiere distintas habilidades, algunas que son propias del desarrollo psico-biológico y otras que dependen del proceso pedagógico social de enseñanza. En este proceso de aprendizaje se combinan el lenguaje oral con las habilidades metalingüísticas y las metodologías de enseñanza. La cultura educacional induce el aprendizaje y da la base para configurar este paradigma.

\section{Referencias}

Akhutina, T. V. (2002). L.S. Vigotsky y A.R. Luria: la formación de la neuropsicología (Y. Solovieva \& L. Quintanar Rojas, Trads.). Revista Española de Neuropsicología, 4, 108-129. Extraído de https://dialnet.unirioja.es/servlet/articulo?codigo=2011215Alegría, J. (1991). Mécanismes spécifiques de la lecture : L'identification des mots écrits [Mecanismos específicos de la lectura: la identificación de palabras escritas]. Les Entretiens Nathan : Lecture. Actes 1, 51-64.

Alegría, J. (2006). Por un enfoque psicolingüístico del aprendizaje de la lectura y sus dificultades-20 años después. Infancia y Aprendizaje, 29, 93-111. https://doi.org/10.1174/021037006775380957

Azcoaga, J. E. (1979). Aprendizaje fisiológico y aprendizaje pedagógico (2a ed.). Buenos Aires, Argentina: El Ateneo.

Benton, A. L. (1971). Introducción a la neuropsicología. Barcelona, España: Fontanella.

Bravo, L. (1980). Teorías sobre la dislexia y su enfoque científico. Santiago, Chile: Editorial Universitaria.

Bravo, L. (1981a). El procesamiento cerebral de la comunicación escrita: un enfoque neuropsicológico. Revista Chilena de Psicología, 4, 81-90.

Bravo, L. (1981b). Special education and learning disabilities in Chile. En L. Tarnopol \& M. Tarnopol (Eds.), Comparative reading and learning difficulties (pp. 257-274). New York, NY: Lexington Books.

Bravo, L. (1982). Dislexia, maduración e integración de funciones cerebrales. Avances en Psicología Clínica Latinoamericana, 1, $111-127$.

Bravo, L. (1990). Dislexia y nivel socioeconómico. Avances en Psicología Clínica Latinoamericana, 8, 43-57.

Bravo, L. (1999). Neuropsicología y psicología cognitiva: convergencia de las investigaciones en dislexias. Avances en Psicología Clínica Latinoamericana, 17, 35-49.

Bravo, L. (2013). Cincuenta años de la psicología en Chile: una perspectiva personal. Psykhe, 22(1), 125-137. https://doi.org/10.7764/psykhe.22.1.631

Bravo, L. (2014). Neuroscience and education: Current state of research on dyslexia. Estudios de Psicología, 35, 1-28. https//doi.org/10.1080/02109395.2014.893648

Bravo, L., Bermeosolo, J. \& Pinto, A. (1987). Dislexia inicial: predictividad de su evolución. Anales de la Facultad de Educación, 10, 115-144.

Bravo, L., Bermeosolo, J. \& Pinto, A. (1988). Evolución e involución del CI en un grupo de disléxicos. Terapia Psicológica, 7(10), 45-50.

Bravo, L., Bermeosolo, J., Pinto, A., Oyarzo, E. \& Morales, M. (1990). Rendimiento neuropsicológico y nivel socioeconómico en niños con retardo lector severo. Revista Chilena de Psicología, 11, 39-46.

Bravo, L. \& Salas, S. (1972). La salud mental en la enseñanza básica. Cuadernos de la Realidad Nacional, 14, $202-208$.

Bravo, L., Villalón, M. \& Orellana, E. (2001). Procesos predictivos del aprendizaje inicial de la lectura en primer año básico. Boletín de Investigación Educacional, 16, 149-160.

Bravo, L., Villalón, M. \& Orellana, E. (2003). Predictividad del rendimiento de la lectura: el segundo año básico. Psykhe, 12(2), 29-36. Extraído de http://www.psykhe.cl/index.php/psykhe/article/view/350/330

Bravo Valdivieso, L. (1973). Trastornos de aprendizaje y de la conducta escolar: psicopatología de la disfunción cerebral infantil. Santiago, Chile: Andrés Bello.

Bravo Valdivieso, L. (1978). La dislexia: enfoques neuropsicológico y psicolingüístico. Revista Chilena de Psicología, 1, 7-16.

Bravo Valdivieso, L. (1985). Dislexias y retraso lector: enfoque neuropsicológico. Madrid, España: Aula XXI/Santillana.

Bravo-Valdivieso, L. (1995). A four year follow-up study of low socioeconomic status, Latin American children with reading dificulties. International Journal of Disability, Development and Education, 42, 189-202. https://doi.org/10.1080/0156655950420302

Bravo Valdivieso, L. (2002). La conciencia fonológica como una zona de desarrollo próximo para el aprendizaje inicial de la lectura. Estudios Pedagógicos, 28, 165-177. https://doi.org/10.4067/S0718-07052002000100010

Bravo Valdivieso, L. (2011). Lenguaje escrito y dislexias: enfoque cognitivo del retardo lector (5a ed.). Santiago Chile: Ediciones Universidad Católica.

Bravo Valdivieso, L. (2013). Lectura inicial y psicología cognitiva ( $3^{\mathrm{a}}$ ed. ampliada). Santiago, Chile: Ediciones Universidad Católica.

Bravo Valdivieso, L. (2016). El aprendizaje del lenguaje escrito y las ciencias de la lectura. Un límite entre la psicología cognitiva, las neurociencias y la educación. Límite, 11(36), 50-59.

Bravo Valdivieso, L. (2017). Neurociencias cognitivas y educación. Revista de Psicología de Arequipa, 7, 117-130. Extraído de http://ucsp.edu.pe/investigacion/psicologia/wp-content/uploads/2017/09/6.-Neurociencias-cognitivas-y-educaci\%C3\%B3n.pdf

Bravo Valdivieso, L. \& Pinto, A. (1980). Los errores específicos en el pronóstico de las dislexias. Revista de Psicología General y Aplicada, $35,1061-1078$.

Bravo-Valdivieso, L. \& Pinto Guevara, A. (1984). Confusiones sensoriales visuales y auditivas en las dislexias. Revista Latinoamericana de Psicología, 16, 213-224. Extraído de http://www.redalyc.org/articulo.oa?id=80516202

Bravo Valdivieso, L. \& Pinto Guevara, A. (1987). Batería de Exploración Verbal para Trastornos de Aprendizaje (B.E.V.T.A): pruebas psicopedagógicas de lenguaje y escritura para evaluar dificultades del aprendizaje escolar. Santiago, Chile: Ministerio de Educación, Centro de Perfeccionamiento Docente. 
Bravo Valdivieso, L., Villalón, M. \& Orellana, E. (2004). Los procesos cognitivos y el aprendizaje de la lectura inicial: diferencias cognitivas entre buenos lectores y lectores deficientes. Estudios Pedagógicos, 30, 7-19. https://doi.org/10.4067/S071807052004000100001

Bravo-Valdivieso, L., Villalón, M. \& Orellana, E. (2006a). Predictibilidad del rendimiento en la lectura: una investigación de seguimiento entre primer y tercer año. Revista Latinoamericana de Psicología, 38, 9-20.

Bravo Valdivieso, L., Villalón, M. \& Orellana, E. (2006b). Diferencias en la predictividad de la lectura entre primer año y cuarto año básicos. Psykhe, 15(1), 3-11. https://doi.org/10.4067/S0718-222820060001000319

Brem, S., Bach, S., Kucian, K., Kujala, J. V., Guttorm, T. K., Martin, E. ... Richardson, U. (2010). Brain sensitivity to print emerges when children learn letter-speech sound correspondences. Proceedings of the National Academy of Science of the United States of America, 107, 7939-7944. https://doi.org//10.1073/pnas.0904402107

Bruer, J. T. (1997). Education and the brain: A bridge too far. Educational Researcher, 26(8), 4-16. https://doi.org/10.3102/0013189X026008004

Condemarín, M. \& Blomquist, M. (1970). La dislexia. Santiago, Chile: Editorial Universitaria.

Damasio, A. (2010). Y el cerebro creó al hombre. ¿Cómo pudo el cerebro generar emociones, sentimientos, ideas y el yo? Barcelona, España: Destino.

Dehaene, S. (2007). Les neurones de la lecture [Las neuronas de la lectura]. Paris, Francia: Odile Jacob.

Dehaene, S. \& Cohen, L. (2007). Cultural recycling of cortical maps. Neuron, 56, 384-398. https://doi.org/10.1016/j.neuron.2007.10.004

Dehaene, S., Cohen, L., Sigman, M. \& Vinckier, F. (2005). The neural code for written words: A proposal. Trends in Cognitives Sciences, 9, 335-341. https://doi.org/10.1016/j.tics.2005.05.004

de Quirós, J. B. \& Della Cella, M. A. (1965). La dislexia en la niñez. Buenos Aires, Argentina: Paidós.

del Río, P. \& Álvarez, A. (2017). Vygotsky and beyond: Horizons for the future of psychology. Estudios de Psicología, 38, 63-114. https//doi.org/10.1080/02109395.2017.1281580

Eccles J. C. (1992). Évolution du cerveau et création de la conscience: A la recherche de la vraie nature de l'homme [Evolución del cerebro y creación de la conciencia: en búsqueda de la verdadera naturaleza del hombre]. Paris, Francia: Fayard.

Fawcett, A. J. \& Nicolson, R. I. (2007). Dyslexia, learning, and pedagogical neuroscience. Developmental Medicine \& Child Neurology, 49, 306-311. https://doi.org/10.1111/j.1469-8749.2007.00306.x

Fletcher, J. M. (2009). Dyslexia: The evolution of a scientific concept. Journal of the International Neuropsychological Society, 15, 501508. https://doi.org/10.1017/S1355617709090900

Galaburda, A. M. (1989). Ordinary and extraordinary brain development: Anatomical variation in developmental dyslexia. Annals of Dyslexia, 39, 65-80. https://doi.org/10.1007/BF02656901

Galaburda, A. M. \& Cestnick, L. (2003). Dislexia del desarrollo. Revista de Neurología, 36(Suplemento 1), S3-S9. Extraído de https://www.neurologia.com/articulo/2003068

Geschwind, N. (1979). Especializaciones del cerebro humano. Investigación y Ciencia (Edición española de Scientific American), 38, 128-138.

Geschwind, N. \& Galaburda, A. M. (1985). Cerebral lateralization. Biological mechanisms, associations, and pathology: I. A hypothesis and a program for research. Archives of Neurology, 42, 428-459. https://doi.org/10.1001/archneur.1985.04060050026008

Geymonat, L. (1970/1984). Historia del pensamiento filosófico y científico (J. Bignozzi, Trad.; Título original: Storia del pensiero filosofico e scientifico). Barcelona, España: Ariel.

Goswami, U. (2004). Neuroscience and education. British Journal of Educational Psychology, 74, 1-14. https://doi.org/10.1348/000709904322848798

Goswami, U. (2014). The neural basis of dyslexia may originate in primary auditory cortex. Brain, 137, 3100-3102. https://doi.org/10.1093/brain/awu296

Habib, M. (1997). Dyslexie : Le cerveau singulier [Dislexia: el cerebro singular]. Marseille, Francia: Solal.

Hynd, G. W., Marshall, R. M. \& Semrud-Clikeman, M. (1991). Developmental dyslexia, neurolinguistic theory and deviations in brain morphology. Reading and Writing, 3, 345-362. https://doi.org/10.1007/BF00354967

Jiménez J. E. (Coord.) (2012). Dislexia en español: prevalencia e indicadores cognitivos, culturales, familiares y biológicos. Madrid, España: Pirámide.

Katzir, T. \& Pare-Blagoev, J. (2006). Applying cognitive neuroscience research to education: The case of literacy. Educational Psychologist, 41, 53-74. https://doi.org/10.1207/s15326985ep4101_6

Kirk, S. A. (1962). Educating exceptional children. Boston, MA: Houghton Mifflin.

Kuhn, T. S. (1996). The structure of scientific revolutions ( $3^{\mathrm{a}}$ ed.). Chicago, IL: The University of Chicago.

Leckman, J. F. \& March, J. S. (2011) Editorial: Developmental neuroscience comes of age. Journal of Child Psychology and Psychiatry, 52, 333-338. https://doi.org/10.1111/j.1469-7610.2011.02378.x

Luria, A. R. (1966). Human brain and psychological processes. New York, NY: Harper \& Row.

Luria, A. R. (1973). The working brain: An introduction to neuropsychology. New York, NY: Basic Books.

Lyon, G. R. (1996). Foundations of neuroanatomy and neuropsychology. En G. R. Lyon \& J. M. Rumsey (Eds.), Neuroimaging: A window to the neurological foundations of learning and behavior in children (pp. 3-24). Baltimore, MD: Paul H. Brookes.

Maurer, U. \& McBride, C. (2017). Neuroscience and literacy acquisition: Introduction to the special section. International Journal of Behavioral Development. Anticipo en línea de la publicación. https://doi.org10.1177/0165025417740435

McCandliss, B. D. (2010). Educational neurosciences: The early years. Proceedings of the National Academy of Science of the United States of America, 107, 8049-8050. https://doi.org/10.1073/pnas.1003431107

Olea, R. (1966). Daño cerebral mínimo. Revista Chilena de Neuro-Psiquiatría, 5, 34-38.

Orellana, E. (1995). Instrumento de evaluación ELEA: fundamentos teóricos y análisis estadístico. Boletín de Investigación Educacional, 10, $163-180$.

Orton, S. T. (1925). "Word blindness" in school children. Archives of Neurology and Psychiatry, 14, 581-615. https://doi.org/10.1001/archneurpsyc.1925.02200170002001

Ozernov-Palchik, O. \& Gaab, N. (2016). Tackling the 'dyslexia paradox': Reading brain and behavior for early markers of developmental dyslexia. WIREs Cognitive Science, 7, 156-176. https://doi.org/10.1002/wcs.1383

Paulesu, E., Frith, U., Snowling, M., Gallagher, A., Morton, J., Frackowiak, R. S. J. \& Frith, C. D. (1996). Is developmental dyslexia a disconnection syndrome? Evidence from PET scanning. Brain, 119, 143-157. https://doi.org/10.1093/brain/119.1.143

Piaget, J. (1968). Le structuralisme [El estructuralismo]. Paris, Francia: Presses Universitaire de France.

Poldrak, R. A. (2001). A structural basis for developmental dyslexia: Evidence from diffusion tensor imaging. En M. Wolf (Ed.), Dyslexia, fluency, and the brain (pp. 213-233). Baltimore, MD: York Press.

Pringle Morgan, W. (1896). A case of congenital word blindness. British Medical Journal, 2, 1378. https://doi.org/10.1136/bmj.2.1871.1378 
Radnitzky, G. (1985). Compatibilité de la vérité et de l'historicité dans les sciences [Compatibilidad de la verdad y la historicidad en la ciencia]. En J. Ladrière, A. Mercier, J. Cohen, E. Scheibe, Y. Gauthier, E. Agazzi ... E. Laszlo, La nature de la vérité scientifique [La naturaleza de la verdad científica] (pp. 133-162). Louvain-la-Neuve, Bélgica: Ciaco.

Rumsey, J. M. (1996). Neuroimaging in developmental dyslexia: A review and conceptualization. En G. R. Lyon \& J. M. Rumsey (Eds.), Neuroimaging: A window to the neurological foundations of learning and behavior in children (pp. 57-77). Baltimore, MD: Paul H. Brookes.

Sandak, R., Mencl, W. E., Frost, S. J. \& Pugh, K. R. (2004). The neurobiologigal basis of skilled and impaired reading: Recent findings and new directions. Scientific Study of Reading, 8, 273-292. https://doi.org/10.1207/s1532799xssr0803_6

Shaywitz, S. E. \& Shaywitz, B. A. (2008). Paying attention to reading: The neurobiology of reading and dislexia. Development and Psychopathology, 20, 1329-1349. https://doi.org/10.1017/S0954579408000631

Shaywitz, B. A., Shaywitz, S. E., Blachman B. A., Pugh, K. R., Fulbright, R. K., Skudarski, P. ... Gore, J. C. (2004). Development of left occipitotemporal systems for skilled reading in children after a phonologically-based intervention. Biological Psychiatry, 55, 926933. https://doi.org/10.1016/j.biopsych.2003.12.019

Sigman, M., Peña, M., Goldin, A. P. \& Ribeiro, S. (2014). Neuroscience and education: Prime time to build the bridge. Nature Neuroscience, 17, 497-502. https://doi.org/10.1038/nn.3672

Simos, P. G., Fletcher, J. M., Bergman, E., Breier, J. I., Foorman, B. R., Catillo, E. M. ... Papanicolaou, A. C. (2002). Dyslexia-specific brain activation profile becomes normal following successful remedial training. Neurology, 58, $1203-1213$. https://doi.org/10.1212/WNL.58.8.1203

Simos, P. G., Fletcher, J. M., Sarkari, S., Billingsley-Marshall, R., Denton, C. A. \& Papanicolaou, A. C. (2007). Intensive instruction affects brain magnetic activity associated with oral word reading in children with persistent reading disabilities. Journal of Learning Disabilities, 40, 37-48. https://doi.org/10.1177/00222194070400010301

Szúcs, D. \& Goswami, U. (2007). Educational neuroscience: Defining a new discipline for the study of mental representations. Mind, Brain, and Education, 1, 114-127. https://doi.org/10.1111/j.1751-228X.2007.00012.x

Tarnopol, L. \& Tarnopol, M. (1981). Comparative reading and learning difficulties. Lexington, MA: Lexington Books.

Vellutino, F. R. (1979). Dyslexia: Theory and research. Cambridge, MA: MIT Press.

Vellutino, F. R., Fletcher, J. M., Snowling, M. J. \& Scanlon, D. M. (2004). Specific reading disability (dislexia): What have we learned in the past four decades? The Journal of Child Psychology and Psychiatry, 45, 2-40. https://doi.org/10.1046/j.0021-9630.2003.00305.x

Vellutino, F. R., Steger, J. A. \& Kandel, G. (1972). Reading disability: An investigation of the perceptual deficit hypothesis. Cortex, 8, 106-118. https://doi.org/10.1016/S0010-9452(72)80030-3

Vergote, A. (1992). Nature, culture, et réalité psychique. Indissociable unité. Revue d'Éthique et de Théologie Morale(Le Supplément, 182-183), 163-193. Extraído de http://theo-psy.fr/WordPress3/wp-content/uploads/2015/11/Vergote-Nature-culture-etr\%C3\%A9alit\%C3\%A9-psychique.pdf

Vygotsky, L. (1934/1995). Pensamiento y lenguaje: cognición del desarrollo humano (J. P. Tosaus Abadía, Trad., Título original: Myshleniye i rech). Barcelona, España: Paidós.

Willingham, D. T. \& Lloyd, J. W. (2007). How educational theories can use neuroscientific data. Mind, Brain, and Education, 1, 140149. https://doi.org/10.1111/j.1751-228X.2007.00014.x

Woodward, W. R. \& Ash, M. G. (Eds.) (1982). The problematic science: Psychology in nineteenth-century thought. New York, NY: Praeger.

Fecha de recepción: Octubre de 2016.

Fecha de aceptación: Marzo de 2018. 